Supporting Information

\title{
Flexible Energy Harvester on a Pacemaker Lead Using Multi-beam Piezoelectric Composite Thin Film
}

Zhe $\mathrm{Xu}^{1 \#}$, Congran Jin ${ }^{1 \#}$, Andrew Cabe ${ }^{2}$, Danny Escobedo2, Nanjing Hao ${ }^{1}$ lan Trase ${ }^{1}$, Andrew B. Closson ${ }^{1}$, , Lin Dong ${ }^{1}$, Yuan Nie ${ }^{1}$, Jim Elliot ${ }^{2}$, Marc D. Feldman ${ }^{2}$, Zi Chen ${ }^{1 *}$ and John X.J. Zhang ${ }^{1 *}$

${ }^{1}$ Thayer School of Engineering, Dartmouth College, Hanover, NH 03755, United States

${ }^{2}$ Division of Cardiology, Department of Medicine, The University of Texas Health Science Center at San Antonio, San Antonio, Texas 78229, USA

\# Authors contributed equally.

* Corresponding author: John X.J. Zhang, John.Zhang@dartmouth.edu

Zi Chen, Zi.Chen@dartmouth.edu 


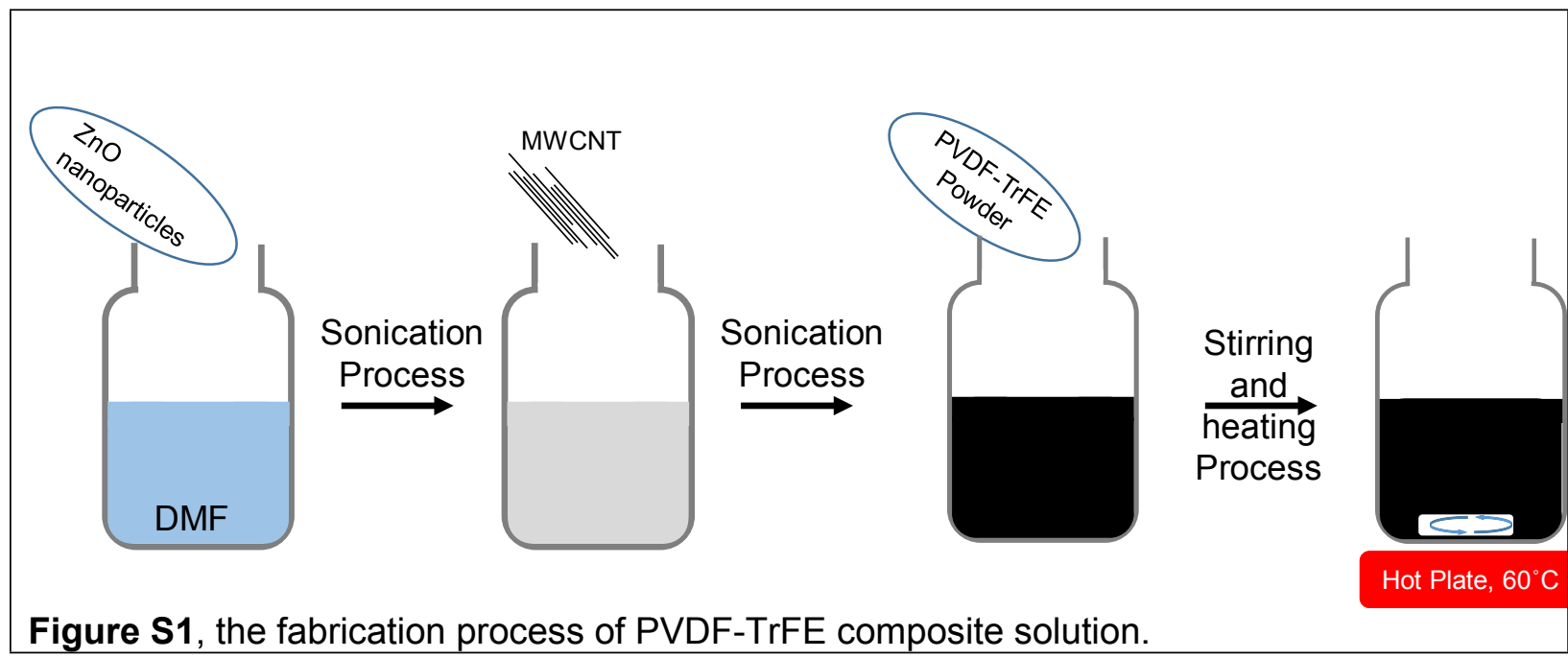




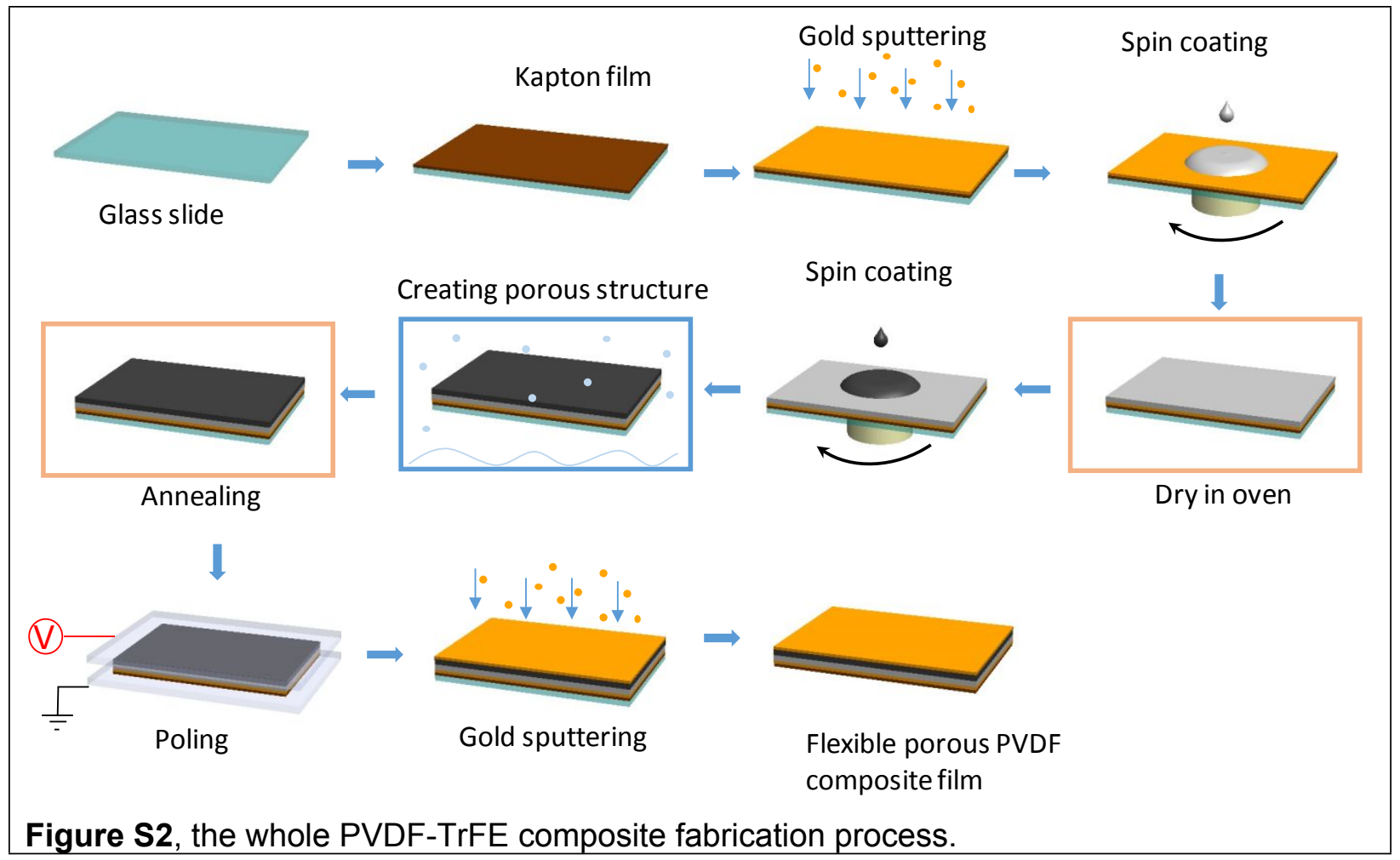




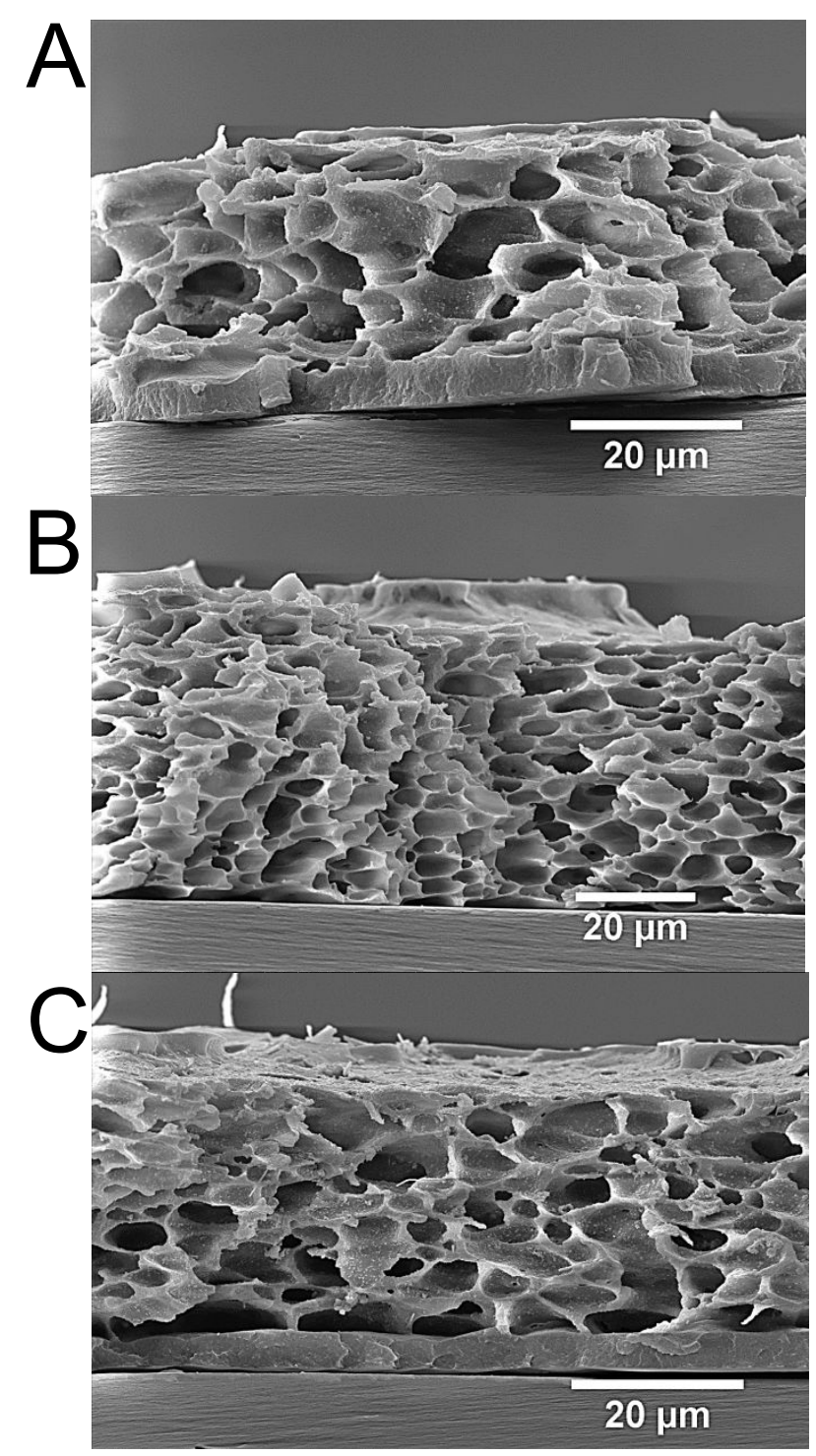

Figure S3, the collection of SEM cross-sectional images from three types of PVDF-TrFE composite films, (A), the composite film with $30 \% \mathrm{ZnO}$, (B) the composite film with $30 \% \mathrm{ZnO}$ and $0.05 \%$ MWCNT, (C), the composite film with $30 \% \mathrm{ZnO}$ and $0.1 \%$ MWCNT 


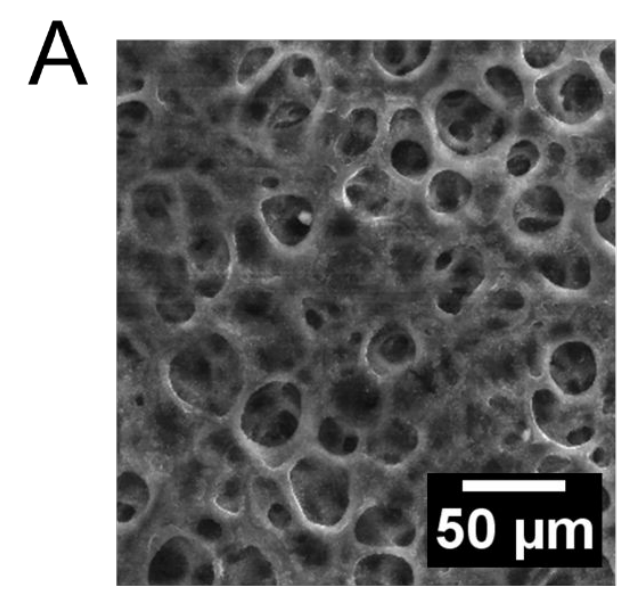

B
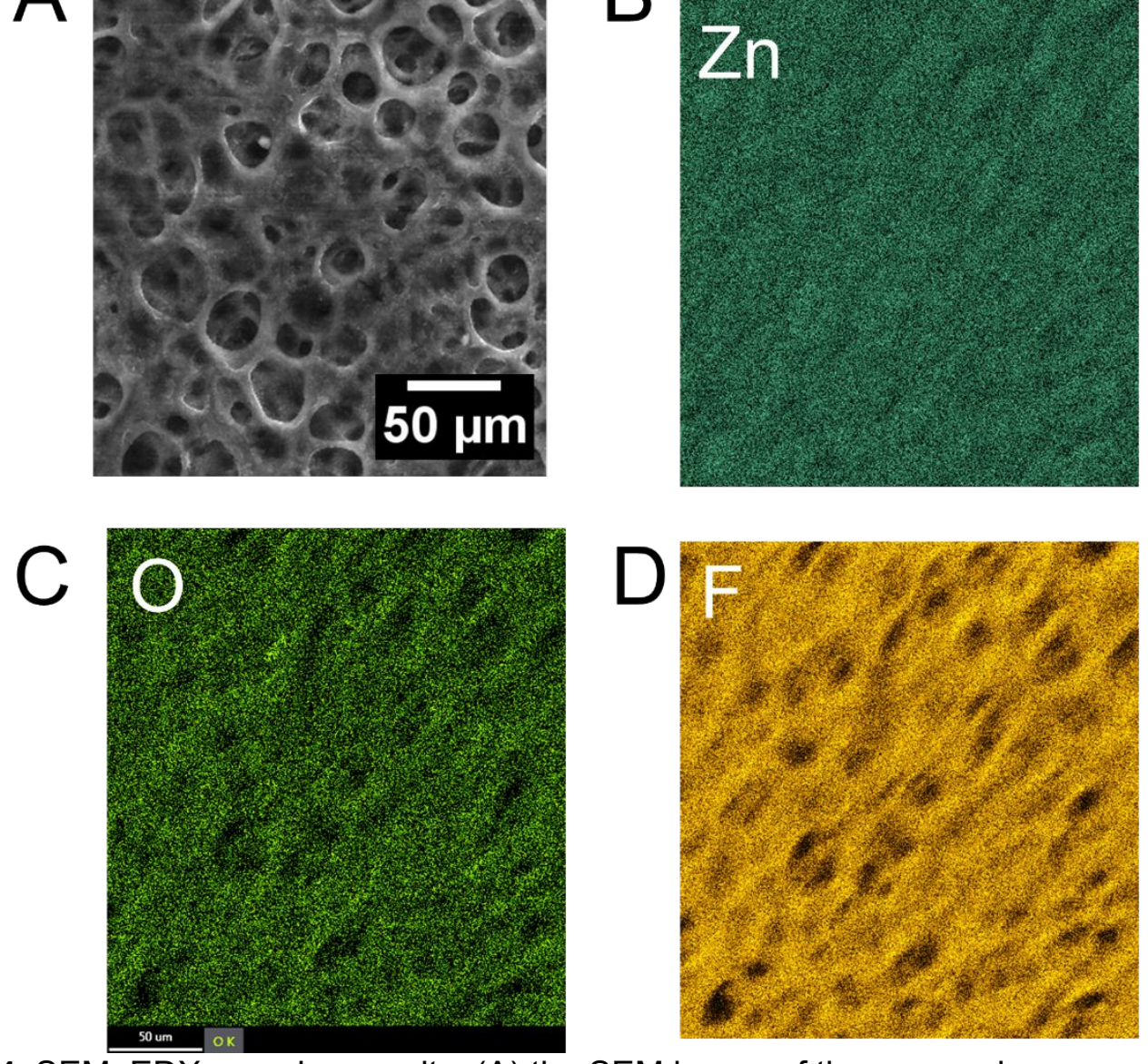

Figure S4, SEM-EDX mapping results. (A) the SEM image of the scanned area, and the chemical mapping of element (B) $\mathrm{Zn},(\mathrm{C}) \mathrm{F}$ and (D) $\mathrm{O}$. 


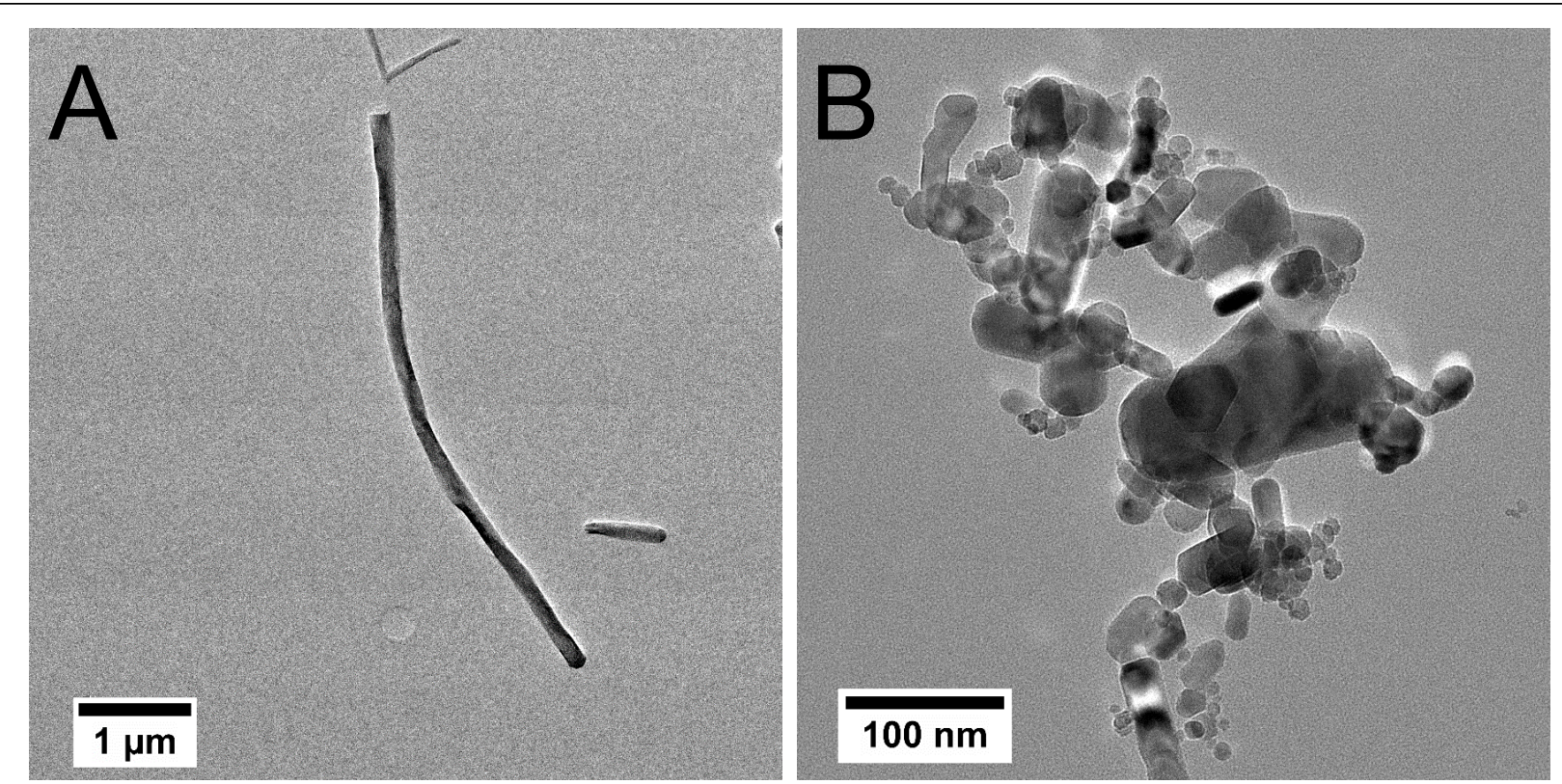

Figure S5, the transmission electron images from two nanomaterials mixed in PVDF-TrFE films, (A) multi-wall carbon nanotube, (B) znic oxide nanoparticles 


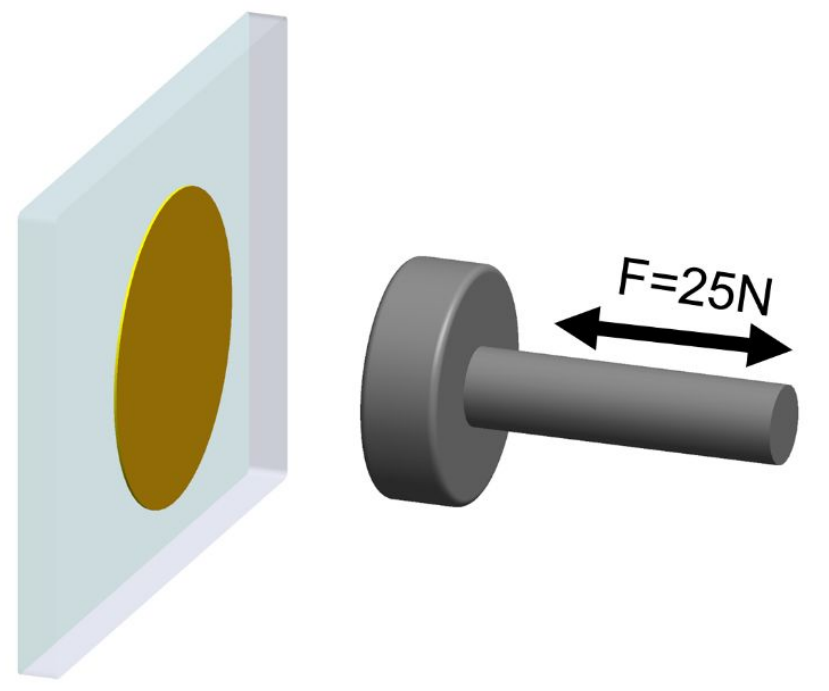

Figure S6, Experimental testing set-up for the impact testing

The impact test setup

To determine the concentration of $\mathrm{ZnO}$ and MWCNT that can produce a larger energy output, we conducted a series of test on the composite film with $10 \%, 30 \%$ and $50 \% \mathrm{ZnO}$ as well as with $0.1 \%$ and $0.05 \%$ MWCNT. The voltage outputs from these samples $(2 \mathrm{~cm}$ in diameter and $\sim 50 \mu \mathrm{m}$ in thickness) are obtained through the following test setup. As shown in Figure S5, a shaker (Model 2060E, The Modal Shop) is used to provide a linear motion with tunable force to the tested composite films. A metal rod with a circular force transducer (Model 208C02, PCB Piezoelectrinics) screwed on its tip is inserted into the shaker. The tested composite film is covered by a piece of $5 \mathrm{~mm} \times 5 \mathrm{~mm} \times 2 \mathrm{~mm}$ PDMS layer to protect it from being damaged by the striking head and is attached to a glass slide. The sample is then fixed to a vertical wall that is fastened on the table. At rest, the tip of the force transducer is about $2 \mathrm{~mm}$ away from the sample. During the test, the shaker is operating at $1 \mathrm{~Hz}$ and $\sim 5.8 \mathrm{~A}$, which exerts $\mathrm{a} \sim 25 \mathrm{~N}$ force on the tested sample. The real-time force information is collected by the force transducer and recorded in LabVIEW (National Instrument) on a desktop. The real-time voltage output is obtained directly by an oscilloscope (TDS 2014B, Tektronix) in a closed circuit. 


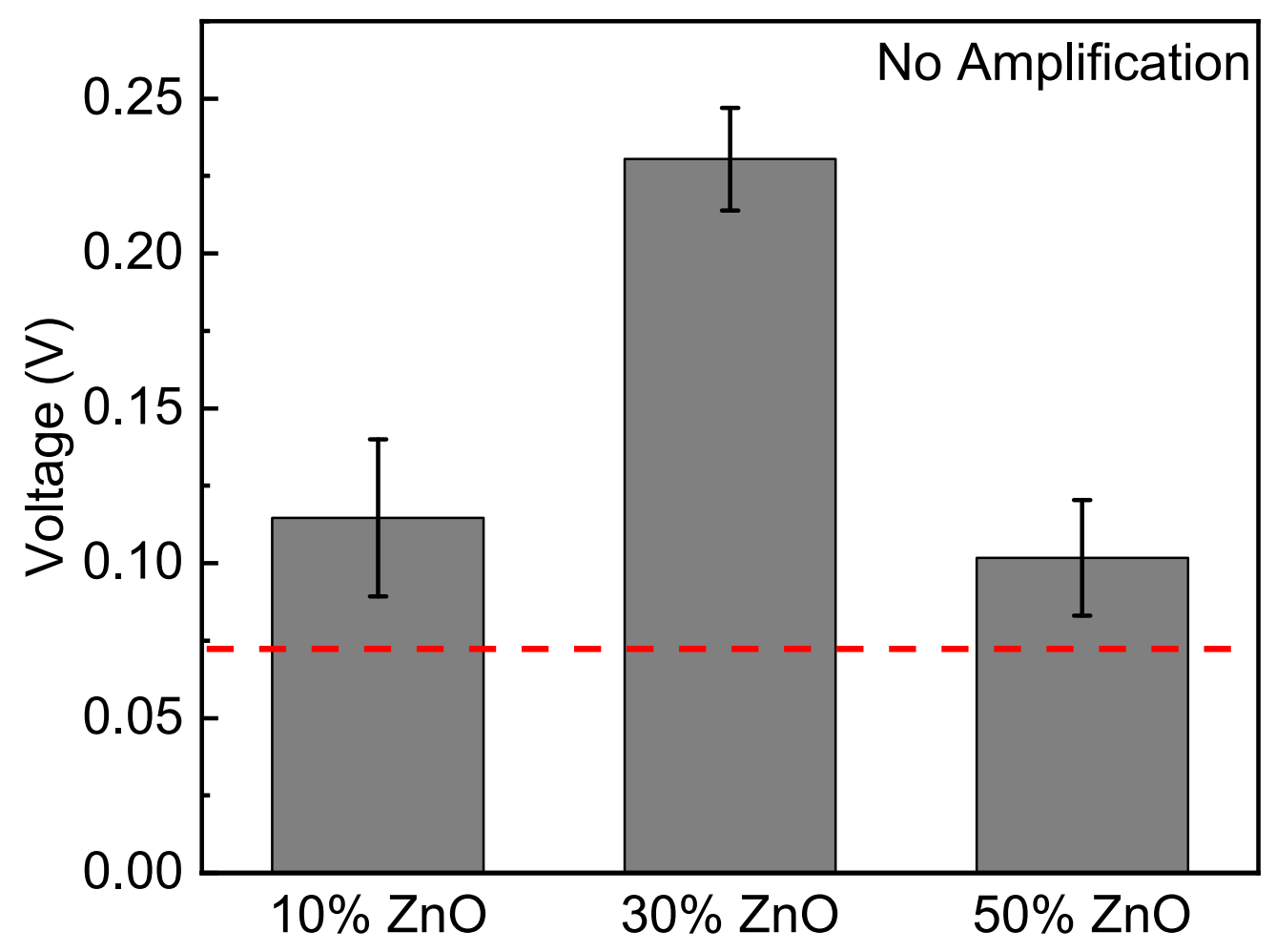

Figure S7, the voltage results comparison between various $\mathrm{ZnO}$ mixed PVDF-TrFE composite film and pure PVDF-TrFE film (red dash line) acquired from impact tests. 


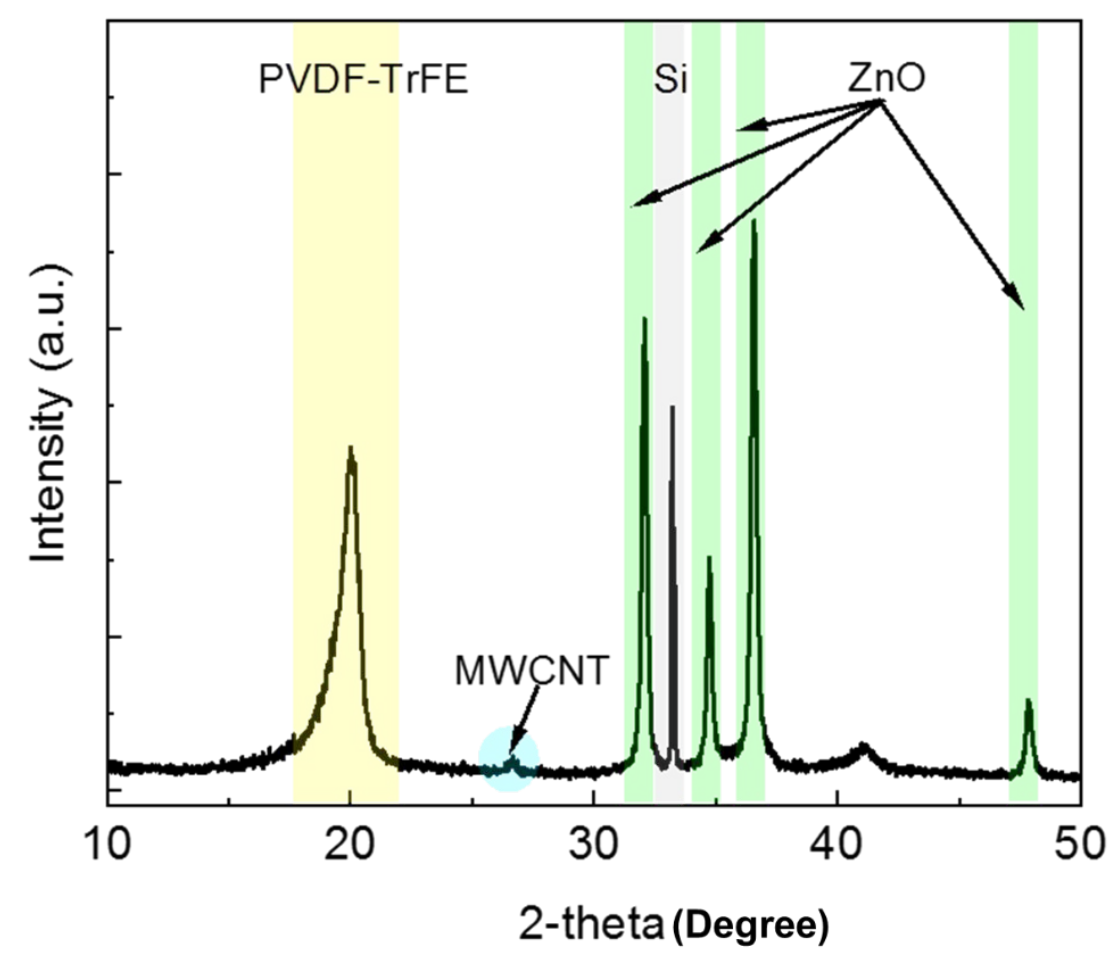

Figure S8, the full range spectrum of XRD acquired from $30 \% \mathrm{ZnO} / 0.1 \% \mathrm{MWCNT}$ PVDFTrFE composite film indicating different components in the composite film. Beta phase of PVDF-TrFE is located at $2 \theta=20^{\circ}$, peaks of $\mathrm{ZnO}$ is located at $2 \theta=32.08^{\circ}, 34.74^{\circ}, 36.58^{\circ}$ and $47.82^{\circ}$, a peak of MWCNT is at $2 \theta=26.68^{\circ}$ 


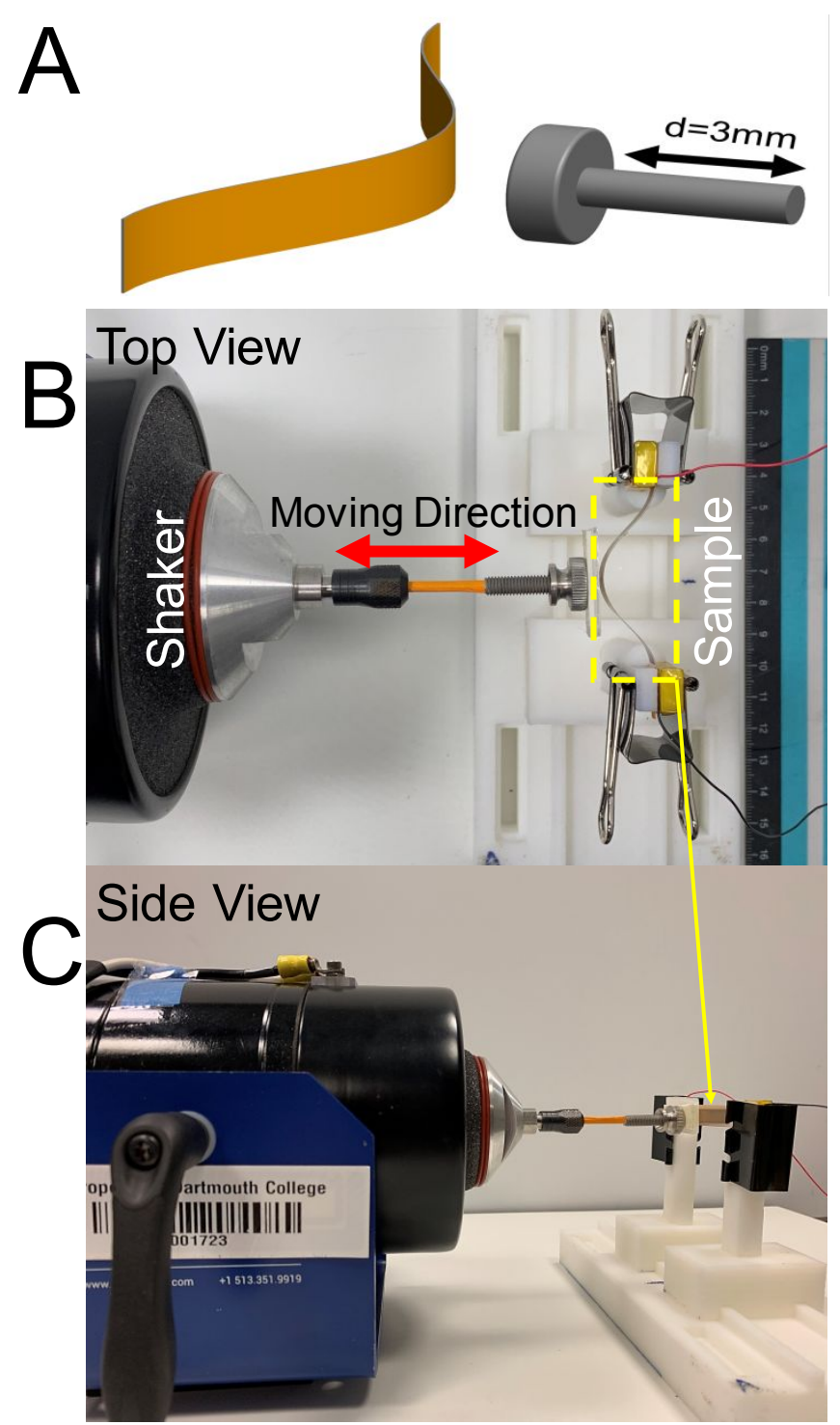

Figure S9, the photography of the low mechanical input testing set-up. 


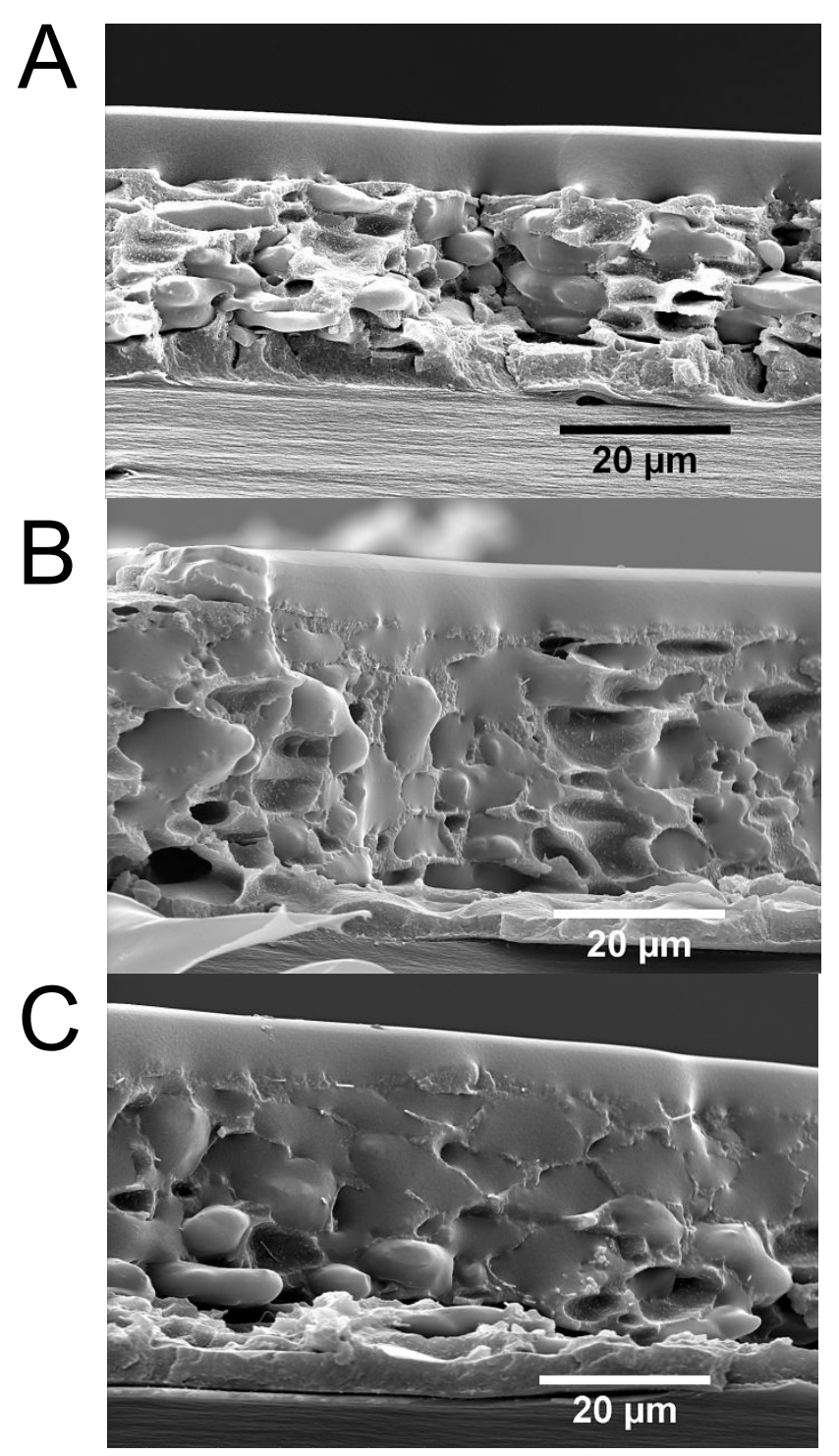

Figure S10, the collection of SEM cross-sectional images from three types of PVDF-TrFE composite films filled with PDMS, (A), the composite film with $30 \% \mathrm{ZnO}$, (B) the composite film with $30 \% \mathrm{ZnO}$ and $0.05 \% \mathrm{MWCNT}$, (C), the composite film with $30 \% \mathrm{ZnO}$ and $0.1 \%$ MWCNT 


\section{Derivation of $\boldsymbol{k}^{2}$ as ratio between clamped and unclamped permittivities}

In the simulation, COMSOL's standard piezoelectric multiphysics module with tetragonal mesh elements is used to describe the PVDF. A $3.5 \mu \mathrm{m} \times 20 \mu \mathrm{m} \times 20 \mu \mathrm{m}$ block of PVDF is generated with a series of $2.2 \mu \mathrm{m}$-radius pores, resulting in a material with a total porosity of $50 \%$. For each infill material, a series of simulations are performed where the block is subjected to an increasing voltage, from $0 \mathrm{~V}$ to $1000 \mathrm{~V}$ in $100 \mathrm{~V}$ increments under open-circuit in the z-direction. In half the simulations, the block is free to move, while in the other half it is fully constrained. For each simulation, the electric displacement on the top surface is measured and used with the voltage to calculate the relative permittivity.

$k^{2}$ is the ratio between the converted energy $U_{C}$ and input energy $U_{I}$. Assume an electric field is applied across a piezoelectric in the z-direction, and assume that the piezoelectric response is mainly also in the $z$-direction (that is, $d_{33} \gg d_{31}$ ). This gives us the relations:

$$
k^{2}=\frac{U_{C}}{U_{I}}, U_{C}=\frac{1}{2} S^{2} E_{C C}, U_{I}=\frac{1}{2} \varepsilon_{U C} E^{2}
$$

Here $S^{2}$ is the strain in the z-direction, $E_{C C}$ is the Young's modulus under a closed circuit, $\varepsilon_{U C}$ is the relative permittivity when the piezoelectric is free to expand and contract, and $E$ is the electric field in the z-direction. We can use the fundamental piezoelectric relation to relate the strain and electric field. When the piezoelectric is free to expand and contract:

$$
S=d E
$$

Here $d$ is the piezoelectric charge constant in the z-directions. Together, these allow us to find a value for $k^{2}$ :

$$
k^{2}=\frac{d^{2} E_{C C}}{\varepsilon_{U C}}
$$

By considering the fundamental equations in the fully constrained limit, we can further simplify our value for $k^{2}$. When the piezeoelectric film is fully constrained, we have the relation:

$$
\varepsilon_{C}=\varepsilon_{U C}-d^{2} E_{C C}
$$

Here $\varepsilon_{C}$ is the relative permittivity when the piezoelectric film is fully constrained. Combining these two equations, we find:

$$
k^{2}=1-\frac{\varepsilon_{C}}{\varepsilon_{U C}}
$$




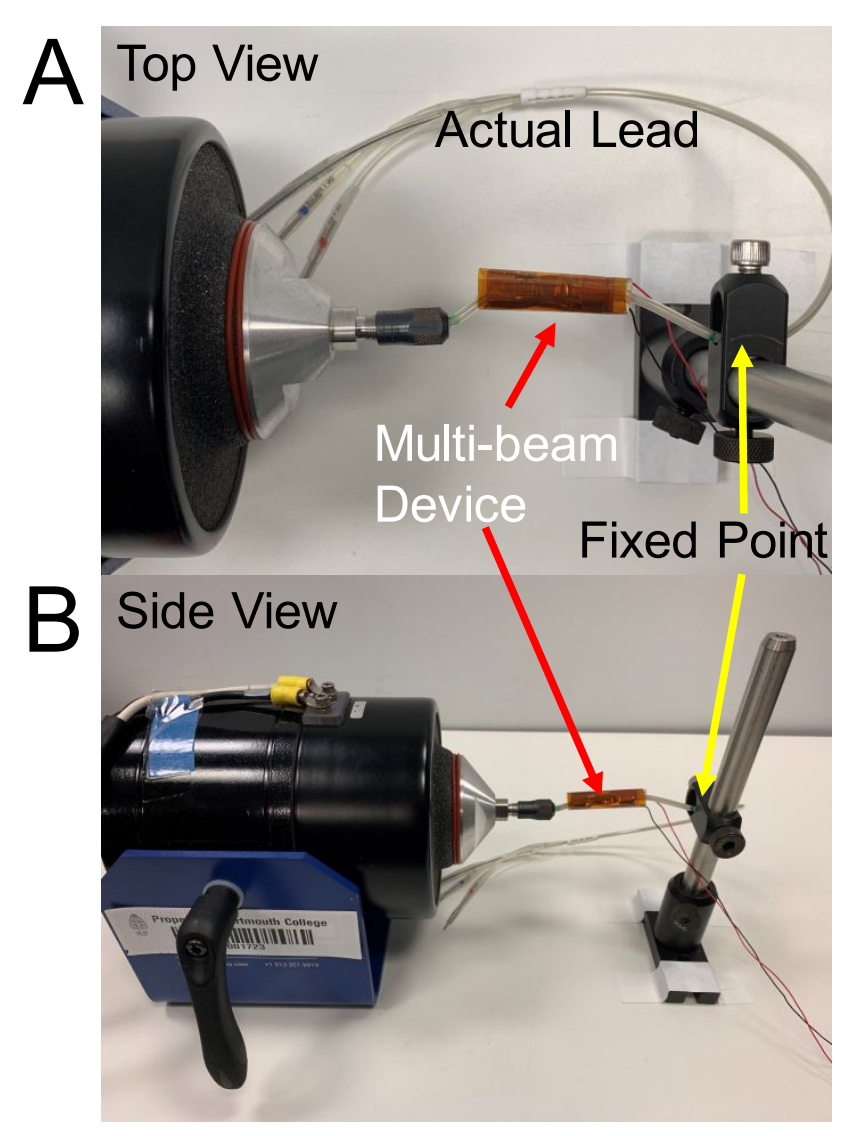

Figure S11, the photography of the testing set-up for simulating the lead bending in human heart and evaluating the electrical properties of the multi-beam device. In the case, the lead we used here is from Medtronic plc with $\sim 2.5 \mathrm{~mm}$ in diameter and the diameter of the housing shell is $10 \mathrm{~mm}$, so the height of buckled beam is $3 \mathrm{~mm}$ calculated by the equation $D=d+2 h$. When the beam array is cut out from a sheet of the composite film, the buckled height of beams is created by the $3 \mathrm{~mm}$ thick spacer. 